Proc. 13th International School on Theoretical Physics: Symmetry and Structural Properties of Condensed Matter

\title{
Information Geometry in the Analysis of Phase Transitions
}

\author{
B. MERA ${ }^{a, b, *}$ \\ ${ }^{a}$ Instituto de Telecomunicações, Lisboa, Portugal \\ ${ }^{b}$ Instituto Superior Técnico, Universidade de Lisboa, Portugal
}

\begin{abstract}
The Uhlmann connection is a mixed state generalization of the Berry connection. The latter has a very important role in the study of topological phases at zero temperature. Closely related, the fidelity is an information theoretical measure of distinguishability between quantum states. We show how one can use the fidelity and the Uhlmann connection to study phase transitions at finite temperature. We apply the analysis to free fermion Hamiltonians in $1 D$ exhibiting symmetry protected topological order at zero temperature and also to the BCS theory of superconductivity. We show how one can study finite-temperature dynamical phase transitions by means of the fidelity and interferometric Loschmidt echoes. Moreover, we explain the physical and mathematical origin of the different behaviour of the two Loschmdit echoes by means of the associated susceptibilities.
\end{abstract}

DOI: 10.12693/APhysPolA.135.1171

PACS/topics: topological phases, information geometry, phase transitions

\section{Introduction}

The classification of phases of matter is a remarkable problem in theoretical physics. The Landau theory of phase transitions $[1,2]$ accurately explains a large class of phases of matter by describing the behaviour of a conveniently chosen local order parameter and associated correlation functions. Topological phases of matter, and in particular, symmetry protected topological phases, such as topological insulators and superconductors [3], constitute a new paradigm in condensed matter physics. Unlike standard phases of matter which are described in terms of local order parameters, these phases of matter are described in terms of global topological invariants, such as the TKNN invariant (named after Thouless, Kohmoto, Nightingale and den Nihjs) [4], mathematically a Chern number associated to a vector bundle over the Brillouin zone, robust against perturbations of the system. An example of such a phase is that of the anomalous Hall insulator [5], which falls into the class of Chern insulators. The topological phases of free fermions were systematically classified through K-theory, by Kitaev [6]; and homotopy theory and Anderson localization, by Schnyder, Ryu, Furusaki and Ludwig [7, 8].

Fidelity is an information theoretical quantity which has been widely used in the study of phase transitions [9-12]. Recently, the problem of the effect of temperature on topological phase transitions has been addressed. For this purpose, in addition to the fidelity, the Uhlmann connection [13, 14], a mixed state generalization of the Berry connection, has been considered and studied in the context of systems exhibiting symmetry protected topological phases at zero temperature [15-17]. The reason for this is that the Berry connection has a remarkable role in the description of topological phases,

*e-mail: bruno.mera@tecnico.ulisboa.pt as it provides a natural connection in the Bloch vector bundle of occupied states over the Brillouin zone, in the presence of translation invariance. The topological invariants can then be built from invariant polynomials on the Berry curvature.

In addition, dynamical phase transitions, which occur when one studies the system after a sudden quench, have been approached, for finite temperatures, through fidelity and information geometry quantities [18].

In this work, we review connection of fidelity to the geometry and gauge theory arising from the phase ambiguity of quantum mechanics. We do this both at the pure state level and mixed state level, motivating the use of the fidelity and the Uhlmann connection to approach the problem of phase transitions in general. Most of the results presented here are based on Refs. [15, 18].

The paper is structured as follows. In Section 2, we recall some geometry and topology arising naturally in the context of quantum mechanics, exemplifying with spin- $\frac{1}{2}$ coherent states and a Chern insulator. In Section 3 , we connect this geometry and topology to the quantum fidelity and introduce the Uhlmann connection. In Section 4, we apply these concepts to phase transitions in free fermion systems, both topological and nontopological. Next, in Section 5, we consider two generalizations of the Loschmidt echo, a figure of merit for dynamical phase transitions, for the case of finite temperatures, one involving the fidelity and the other an interferometric quantity. We discuss its physical and mathematical origins by studying the associated susceptibilities. Finally, in Section 6, we present some conclusions.

\section{Geometry, topology, and gauge theory in Quantum Mechanics}

A pure state in quantum mechanics is a ray, i.e., it is only defined modulo multiplication by a non-zero scalar. In other words, given a vector $|\psi\rangle$ in the Hilbert space $\mathcal{H}$, we are instructed to identify $|\psi\rangle \sim \lambda|\psi\rangle$, with $\lambda \in \mathbb{C}^{\times}$. 
This means that a state is a one-dimensional subspace of $\mathcal{H}$ and the set of all states is the quotient space $\mathbb{P} \mathcal{H}=(\mathcal{H}-\{0\}) / \mathbb{C}^{\times}$, the projective space associated to $\mathcal{H}$. We could restrict ourselves to normalized states, i.e., $S \mathcal{H}=\{|\psi\rangle \in \mathcal{H}:\langle\psi \mid \psi\rangle=1\}$ and then one realizes that a state is a normalized vector modulo a phase, i.e., $\mathbb{P H}=S \mathcal{H} / \mathrm{U}(1)$. We, therefore, have a $\mathrm{U}(1)$-gauge degree of freedom in specifying a state. The gauge invariant information is uniquely specified by the orthogonal projector

$$
P=|\psi\rangle\left\langle\psi\left|=\mathrm{e}^{\mathrm{i} \phi}\right| \psi\right\rangle\langle\psi| \mathrm{e}^{-\mathrm{i} \phi}, \text { for all } \mathrm{e}^{\mathrm{i} \phi} \in \mathrm{U} .
$$

More generally, we can think of the space of $k$-dimensional subspaces of $\mathcal{H}$, the Grassmannian of $k$ planes in $\mathcal{H}, \operatorname{Gr}_{k}(\mathcal{H})$. A $k$-dimensional subspace $V \subset \mathcal{H}$ is specified by giving an orthonormal basis $\left\{\left|\psi_{i}\right\rangle, i=\right.$ $1, \ldots, k\}$. However, if $U=\left[u^{i}{ }_{j}\right]_{1 \leq i, j \leq k} \in \mathrm{U}(k)$ then

$$
\left|\psi_{i}^{\prime}\right\rangle=\sum_{j} u_{i}^{j}\left|\psi_{j}\right\rangle, \quad i=1, \ldots, k
$$

is another orthonormal basis for $V$ and, hence, we have a $\mathrm{U}(k)$-gauge degree of freedom in defining $V \subset \mathcal{H}$ through an orthonormal basis. A gauge invariant description of this subspace $V$ is specified by the projector

$$
\begin{aligned}
& P=\sum_{i=1}^{k}\left|\psi_{i}\right\rangle\left\langle\psi_{i}\left|=\sum_{i, j, l=1}^{k} u_{i}^{j}\right| \psi_{j}\right\rangle\left\langle\psi_{l}\right| \bar{u}_{i}^{l}, \\
& \text { for all } U=\left[u^{i}{ }_{j}\right]_{1 \leq i, j \leq k} \in \mathrm{U}(k) .
\end{aligned}
$$

This unitary gauge ambiguity in specifying a state or a subspace has important physical consequences. Moreover, associated with this gauge ambiguity there is a natural gauge field which arises from the Hilbert space structure. Specifically, the $\mathfrak{u}(1)$-valued 1 -form

$$
A=\langle\psi|\mathrm{d}| \psi\rangle, \text { with } \bar{A}=-A,
$$

defines the $\mathrm{U}(1)$-Berry connection and the $\mathfrak{u}(k)$-valued 1 -form

$$
A=\left[\left\langle\psi_{i}|\mathrm{~d}| \psi_{j}\right\rangle\right]_{1 \leq i, j \leq k}, \text { with } A^{\dagger}=-A,
$$

defines the $\mathrm{U}(k)$-Berry connection.

The Abelian Berry connection allows us to split the tangent spaces of $S \mathcal{H}$ into vertical directions, i.e., those which do not change the physical state, and horizontal directions, those that effectively change the physical state. Since $|\psi\rangle$ defines the same state as $\mathrm{e}^{\mathrm{i} \phi}|\psi\rangle=$ $(1+\mathrm{i} \phi+\ldots)|\psi\rangle$, the vertical directions are immediately seen to be those that are a purely imaginary multiple of the state. The horizontal directions are simply the orthogonal complement (induced by the inner product). Namely, given a curve of normalized vectors in $\mathcal{H}$, $\{|\psi(t)\rangle: t \in[0,1]\}$, we can write

$$
\frac{\mathrm{d}|\psi(t)\rangle}{\mathrm{d} t}=\underbrace{\overbrace{\left\langle(t)\left|\frac{\mathrm{d}}{\mathrm{d} t}\right| \psi(t)\right\rangle} \overbrace{}^{A(\mathrm{~d}|\psi\rangle / \mathrm{d} t)}|\psi(t)\rangle}_{\text {Vertical }}
$$

$$
+\underbrace{\frac{\mathrm{d}|\psi(t)\rangle}{\mathrm{d} t}-\left\langle\psi(t)\left|\frac{\mathrm{d}}{\mathrm{d} t}\right| \psi(t)\right\rangle|\psi(t)\rangle}_{\text {Horizontal }} \text {, for all } t \in[0,1]
$$

With this splitting of the tangent spaces one can define parallel transportation along a curve of states. The result is that, under parallel transport,

$$
|\psi(0)\rangle \longrightarrow \mathrm{e}^{-\int_{0}^{1} \mathrm{~d} t A(\mathrm{~d}|\psi\rangle / \mathrm{d} t)}|\psi(1)\rangle .
$$

The obstruction to integrability, in the sense of the Frobenius theorem [19], of the horizontal distribution, i.e., the collection of the horizontal spaces, associated to the Berry connection, is given by the Berry curvature twoform

$$
F=\mathrm{d} A=\operatorname{Tr}(P \mathrm{~d} P \wedge \mathrm{d} P) .
$$

The Berry curvature is also associated to the parallel transport along an infinitesimal loop.

Given this splitting of the tangent spaces, we can then define a Riemannian metric on $\mathbb{P H}$ by the gauge-invariant formula

$$
\begin{aligned}
& \mathrm{d} s^{2}=\| \mathrm{d}|\psi\rangle-\langle\psi|\mathrm{d}| \psi\rangle|\psi\rangle \|^{2}= \\
& \quad\langle\mathrm{d} \psi|(I-|\psi\rangle\langle\psi|)| \mathrm{d} \psi\rangle=\operatorname{Tr}(P \mathrm{~d} P \mathrm{~d} P),
\end{aligned}
$$

which is the celebrated Fubini-Study metric in $\mathbb{P} \mathcal{H}$, also known as the quantum metric or the Bures metric. The quantum metric and the Berry curvature are the real and imaginary parts of a Hermitian tensor - the quantum geometric tensor.

For more details on the fiber bundles and associated structures considered here, presented from a physical point of view, the reader is referred to Refs. [20, 21]. For a more detailed mathematical exposition on the theory of fiber bundles and differential forms, see Refs. [19, 22].

$$
\begin{aligned}
& 2.1 \mathcal{H}=\mathbb{C}^{2}: \text { Spin- } \frac{1}{2} \text { coherent states } \\
& \text { and topological phases of matter }
\end{aligned}
$$

When $\mathcal{H}=\mathbb{C}^{2}$, i.e., for the case of a spin- $\frac{1}{2}$ particle, we have that the space of states is the Riemann sphere $\mathbb{C} P^{1} \cong \mathbb{C} \cup\{\infty\} \cong S^{2}$, also known as the Bloch sphere in the physics literature. If we write $\boldsymbol{n}$ for a threedimensional unit vector parametrized by spherical coordinates $(\theta, \phi)$,

$$
\boldsymbol{n}=\left(x^{1}, x^{2}, x^{3}\right)=(\sin \theta \cos \phi, \sin \theta \sin \phi, \cos \theta),
$$

then we can obtain a representative of the state associated to it, by the formula

$$
|\boldsymbol{n}\rangle \equiv|z\rangle=\frac{1}{\left(1+|z|^{2}\right)^{1 / 2}}\left(\begin{array}{l}
1 \\
z
\end{array}\right)
$$

where

$$
z=\frac{x^{1}+\mathrm{i} x^{2}}{1+x^{3}}=\mathrm{e}^{\mathrm{i} \phi} \tan \left(\frac{\theta}{2}\right)
$$

is the complex coordinate associated to stereographic projection with respect to the south pole of the sphere. The state $|z\rangle$ is the so-called spin- $\frac{1}{2}$ coherent state. Notice that this representative is only valid for $x^{3} \neq-1$, i.e., everywhere on $S^{2}$ except at the south pole, see Fig. 1. Mathematically, $|z\rangle$ defines a local section of a 
non-trivial line bundle over $S^{2}$, whose fibre over a state is the state/ray itself - the monopole bundle. To describe the full space of states, we need another trivialization valid in a neighbourhood of the south pole. If we take $w=1 / z$, then

$$
|z\rangle=\frac{z}{|z|} \times \frac{1}{\left(1+|w|^{2}\right)^{1 / 2}}\left(\begin{array}{c}
w \\
1
\end{array}\right) .
$$

Notice that the coordinate $w$ corresponds to stereographic projection with respect to the north pole and $w=1 / z$ is the complex coordinate change which endows $S^{2}$ with the structure of a complex manifold. Moreover,

$$
\frac{1}{\left(1+|w|^{2}\right)^{1 / 2}}\left(\begin{array}{c}
w \\
1
\end{array}\right)
$$

represents any quantum state for $w \neq \infty$, i.e., all of them except the one corresponding to the north pole. The relation between the two descriptions of the states is captured by the gauge transformation $z /|z|$ defined in the sphere minus the poles. The Berry connection gives rise to a connection in the monopole bundle given by, in the gauge associated to $|z\rangle$,

$$
A=\langle z|\mathrm{~d}| z\rangle=\frac{1}{2} \frac{\bar{z} \mathrm{~d} z-z \mathrm{~d} \bar{z}}{\left(1+|z|^{2}\right)} .
$$

The metric in the space of states is the Fubini-Study metric:

$$
\mathrm{d} s^{2}=\frac{\mathrm{d} z \mathrm{~d} \bar{z}}{\left(1+|z|^{2}\right)^{2}}=\frac{1}{4}\left(\mathrm{~d} \theta^{2}+\sin ^{2} \theta \mathrm{d} \phi^{2}\right),
$$

which shows that the Bloch sphere has radius $1 / 2$.

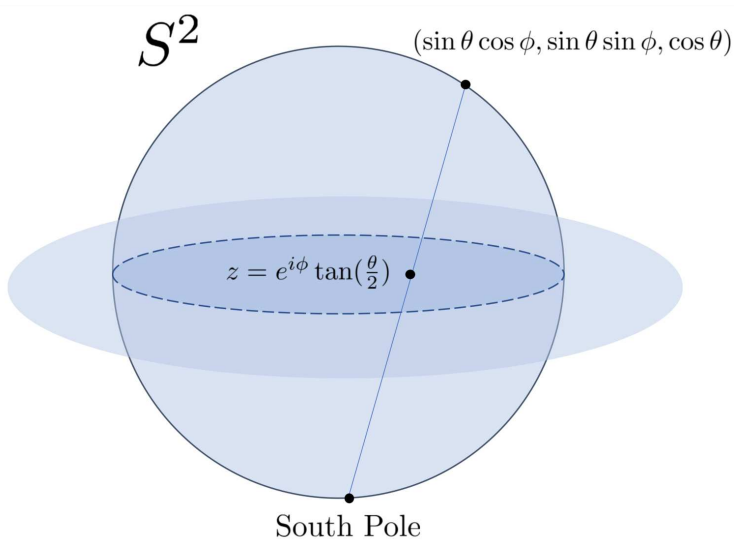

Fig. 1. The space of states of a two-dimensional quantum system is a sphere $S^{2}$. A complex coordinate is obtained by stereographic projection.

The Berry curvature is given by

$$
\begin{gathered}
F=\mathrm{d} A=\frac{\mathrm{d} \bar{z} \wedge \mathrm{d} z}{\left(1+|z|^{2}\right)^{2}}=\frac{\mathrm{i}}{2} \sin \theta \mathrm{d} \theta \wedge \mathrm{d} \phi= \\
\frac{\mathrm{i}}{2} \boldsymbol{n} \cdot(\mathrm{d} \boldsymbol{n} \times \mathrm{d} \boldsymbol{n})=\frac{\mathrm{i}}{4} \frac{\varepsilon_{i j k} x^{i} \mathrm{~d} x^{j} \wedge \mathrm{d} x^{k}}{\|\boldsymbol{x}\|^{3}},
\end{gathered}
$$

which corresponds to a "magnetic field" $\boldsymbol{B}=$ $-(1 / 4 \pi) \boldsymbol{x} /\|\boldsymbol{x}\|^{3}$ of a monopole of charge -1 sitting at the origin of $\mathbb{R}^{3}$. In fact, the integer quantity

$$
\int_{S^{2}} \frac{\mathrm{i} F}{2 \pi}=-1 \in \mathbb{Z}
$$

is a topological invariant known as the 1st Chern number, which measures the obstruction of this monopole bundle to being topologically trivial, i.e., isomorphic, as a vector bundle, to $S^{2} \times \mathbb{C}$.

Next, suppose we have a gapped $2 D$ charge symmetric fermion quadratic lattice Hamiltonian with translation invariance described by

$$
H(\boldsymbol{k})=\boldsymbol{d}(\boldsymbol{k}) \cdot \boldsymbol{\sigma},
$$

where $\boldsymbol{\sigma}=\left(\sigma_{1}, \sigma_{2}, \sigma_{2}\right)$ are the Pauli matrices, $\boldsymbol{k}$ is the quasi-momentum which lives in the first Brillouin zone (BZ). The first Brillouin zone is, topologically, a torus, since we are instructed to identify momenta which differ by reciprocal lattice vectors. The gap condition can be stated simply as $\boldsymbol{d}(\boldsymbol{k}) \neq 0$, for all $\boldsymbol{k} \in \mathrm{BZ}$. The groundstate is obtained by filling the lowest energy band

$$
L_{\boldsymbol{k}}:=\left\{|\psi\rangle \in \mathbb{C}^{2}: H(\boldsymbol{k})|\psi\rangle=-|\boldsymbol{d}(\boldsymbol{k})||\psi\rangle\right\}, \quad \boldsymbol{k} \in \mathrm{BZ} .
$$

The (disjoint) union of these one-dimensional subspaces is the (occupied) Bloch bundle $L=\coprod_{\boldsymbol{k} \in \mathrm{BZ}} L_{\boldsymbol{k}} \rightarrow \mathrm{BZ} \cong$ $T^{2}$. Take $\boldsymbol{n}(\boldsymbol{k}) \equiv \boldsymbol{d}(\boldsymbol{k}) /|\boldsymbol{d}(\boldsymbol{k})|$, which is well-defined because of the gap condition. Then, the spin- $\frac{1}{2}$ coherent state $|-\boldsymbol{n}(\boldsymbol{k})\rangle \equiv|-1 / \bar{z}(\boldsymbol{k})\rangle$, where $z(\boldsymbol{k})$ is obtained by stereographic projection as before, is a representative for $L_{\boldsymbol{k}}$. Mathematically, $L$ is the pullback bundle of the monopole bundle over the Riemann sphere by the map $\Phi:$ BZ $\ni \boldsymbol{k} \mapsto-\boldsymbol{n}(\boldsymbol{k}) \in S^{2}$. As a consequence, the first Chern number of $L$ is the degree or winding number of $\Phi$ :

$$
\begin{aligned}
& \int_{\mathrm{BZ}} \frac{\mathrm{i} F}{2 \pi}= \\
& \frac{1}{4 \pi} \int_{\mathrm{BZ}} \frac{\boldsymbol{d}(\boldsymbol{k}) \cdot\left(\frac{\partial \boldsymbol{d}(\boldsymbol{k})}{\partial k_{x}} \times \frac{\partial \boldsymbol{d}(\boldsymbol{k})}{\partial k_{y}}\right)}{\|\boldsymbol{d}(\boldsymbol{k})\|^{3}} \mathrm{~d}^{2} k \equiv \operatorname{deg} \Phi .
\end{aligned}
$$

Moreover, the transverse Hall conductivity is precisely given by $q^{2} /(2 \pi)$ times the Chern number $[4,23]$, in natural units, where $q$ is the charge of the fermions in the theory. This shows an intricate relation between the geometry and topology of the states of the system and the physical response to an external gauge field.

\section{Fidelity, Uhlmann connection and phase transitions}

Fidelity is an information theoretical notion. It is a measure of distinguishability between two quantum states. Whenever a phase transition occurs, the state of the system changes dramatically and fidelity captures this change. For pure states $|\psi\rangle,\left|\psi^{\prime}\right\rangle \in \mathcal{H}$,

$$
F\left(|\psi\rangle,\left|\psi^{\prime}\right\rangle\right)=\left|\left\langle\psi \mid \psi^{\prime}\right\rangle\right| \text {. }
$$

If $|\psi(g)\rangle$ is a groundstate of a family of Hamiltonians $\{H(g)\}$, the quantity

$$
F(|\psi(g)\rangle,|\psi(g+\delta g)\rangle) \text {, with }|\delta g|<<1,
$$


will generically be close to 1 . When $g$ reaches a point of phase transition, $F$ will have a sudden drop. The fidelity analysis provides a method to probe quantum phase transitions.

Associated to the fidelity, one can define the fidelity susceptibility:

$$
\chi(g)=-\left.\frac{1}{N} \frac{\partial^{2} \log F(|\psi(g)\rangle,|\psi(g+h)\rangle)}{\partial h^{2}}\right|_{h=0},
$$

where $N$ is the number of degrees of freedom of the system, which diverges at the critical points of quantum phase transition in the thermodynamic limit $N \rightarrow \infty$. We will see that this object is intrinsically geometric.

\subsection{Relation to the Bures distance,} the Berry phase and the Bures metric

Let $\left[g_{\text {initial }}, g_{\text {final }}\right] \ni g \mapsto|\psi(g)\rangle \in \mathcal{H}$ be a curve and $\left\{\left|\psi_{i}\right\rangle=\left|\psi\left(g_{i}\right)\right\rangle: i=1, \ldots, M\right\}$ be a discretization of the curve. Then,

$$
\begin{aligned}
& \|\left|\psi_{i+1}\right\rangle-\left|\psi_{i}\right\rangle \|^{2}=2\left(1-\operatorname{Re}\left\langle\psi_{i+1} \mid \psi_{i}\right\rangle\right) \\
& \quad \geq 2\left(1-F\left(\left|\psi_{i+1}\right\rangle,\left|\psi_{i}\right\rangle\right)\right) \equiv d_{\text {Bures }}^{2}\left(\left|\psi_{i+1}\right\rangle,\left|\psi_{i}\right\rangle\right),
\end{aligned}
$$

where $\|\cdot\|$ is the Hilbert space norm induced by the Hermitian inner product and $d_{\text {Bures }}(\cdot, \cdot)$ is the so-called Bures distance. Choosing different representatives for the quantum states $\left|\widetilde{\psi}_{i}\right\rangle=\mathrm{e}^{\mathrm{i} \alpha_{i}}\left|\psi_{i}\right\rangle$ does not change the fidelity nor the Bures distance, i.e., they are gauge invariant. In particular, we can choose them so that the inequality is saturated for each $i$. When $M \rightarrow \infty$,

$$
\lim _{M \rightarrow \infty} \mathrm{e}^{\mathrm{i} \alpha_{M}}=\exp \left(-\int_{g_{\text {initial }}}^{g_{\text {final }}}\left\langle\psi(g)\left|\frac{\mathrm{d}}{\mathrm{d} g}\right| \psi(g)\right\rangle \mathrm{d} g\right),
$$

which is the Berry phase obtained by parallel transportation of $|\psi(0)\rangle$ along the curve of quantum states.

Additionally,

$$
\begin{aligned}
& F\left(\left|\psi_{i+1}\right\rangle,\left|\psi_{i}\right\rangle\right)=\left|\left\langle\psi_{i+1} \mid \psi_{i}\right\rangle\right| \\
& \approx 1-\frac{1}{2} \| \frac{\mathrm{d}|\psi(g)\rangle}{\mathrm{d} g}-\left\langle\psi(g)\left|\frac{\mathrm{d}}{\mathrm{d} g}\right| \psi(g)\right\rangle|\psi(g)\rangle \|^{2}\left(g_{i+1}-g_{i}\right)^{2} \\
& \approx \exp \left(-\frac{1}{2} \| \frac{\mathrm{d}|\psi(g)\rangle}{\mathrm{d} g}-\left\langle\psi(g)\left|\frac{\mathrm{d}}{\mathrm{d} g}\right| \psi(g)\right\rangle|\psi(g)\rangle \|^{2} \delta g^{2}\right) \\
& \equiv \exp \left(-\frac{1}{2} N \chi(g) \delta g^{2}\right) .
\end{aligned}
$$

This last equation shows that fidelity susceptibility is the pullback of the quantum metric by the map $g \mapsto P(g)=$ $|\psi(g)\rangle\langle\psi(g)|$.

The susceptibility can also be written in terms of the projector $P(g)=|\psi(g)\rangle\left\langle\psi(g)|\equiv| \psi_{0}(g)\right\rangle\left\langle\psi_{0}(g)\right|$ and its complement $Q(g)=\sum_{i \neq 0}\left|\psi_{i}(g)\right\rangle\left\langle\psi_{i}(g)\right|$,

$$
\chi(g)=\frac{1}{N} \operatorname{Tr}\left(P(g) \frac{\partial P(g)}{\partial g} Q(g) \frac{\partial P(g)}{\partial g}\right) .
$$

If furthermore $|\psi(g)\rangle$ is the groundstate of a family of Hamiltonians $H(g)$, then we can write the fidelity susceptibility as a sum over excited states:

$$
\chi(g)=\frac{1}{N} \sum_{i \neq 0} \frac{\left|\left\langle\psi_{0}(g)\left|\frac{\partial H(g)}{\partial g}\right| \psi_{i}(g)\right\rangle\right|^{2}}{\left(E_{0}(g)-E_{i}(g)\right)^{2}},
$$

where the $E_{i}(g)$ 's denote the energies associated to the states $\left|\psi_{i}(g)\right\rangle$. The last sum over states indicates how the gap closing points lead to divergences.

\subsection{Fidelity, Bures distance and Uhlmann connection}

The fidelity generalizes to mixed states $\rho$ and $\rho^{\prime}$ through the formula:

$$
F\left(\rho, \rho^{\prime}\right)=\operatorname{Tr}\left(\sqrt{\sqrt{\rho} \rho^{\prime} \sqrt{\rho}}\right) .
$$

Associated to it are the Bures distance, and its infinitesimal version, the Bures metric, and the Uhlmann connection. The Uhlmann connection generalizes the Berry connection. For families of fixed rank projectors, it reduces to the non-Abelian Berry connection.

The geometric point of view here is that if we consider the space of fixed rank $k$ density matrices over $\mathcal{H}$, considered to be finite dimensional, say $\mathcal{H}=\mathbb{C}^{n}$, there is a principal $\mathrm{U}(k)$-bundle whose fiber at $\rho$ is the set of purifications of $\rho$. The later can be seen as the set of $n \times k$ matrices $W$, such that

$$
W W^{\dagger}=\rho .
$$

The right action of $\mathrm{U}(k), W \mapsto W \cdot U$, with $U \in \mathrm{U}(k)$, clearly preserves the fibre. The tangent bundle to the space of all purifications of density matrices of fixed rank $k$ comes endowed with a Riemannian metric induced by the Hilbert-Schmidt, or Frobenius inner product. This allows us to define a horizontal distribution as before and, hence, parallel transport along curves of density matrices - the resulting connection is called the Uhlmann connection.

To capture the non-triviality of the Uhlmann gauge field, we can define the quantity

$$
\begin{gathered}
\Delta\left(\rho, \rho^{\prime}\right):=F\left(\rho, \rho^{\prime}\right)-\operatorname{Tr}\left(\sqrt{\rho} \sqrt{\rho^{\prime}}\right)= \\
\operatorname{Tr}\left(\sqrt{\sqrt{\rho} \rho^{\prime} \sqrt{\rho}}\right)-\operatorname{Tr}\left(\sqrt{\rho} \sqrt{\rho^{\prime}}\right) .
\end{gathered}
$$

The reason for this is that for a curve of maximal rank density operators, $t \mapsto \rho(t)$,

$$
\begin{aligned}
& \Delta(\rho(t), \rho(t+\delta t))= \\
& \quad \operatorname{Tr}[|\sqrt{\rho(t+\delta t)} \sqrt{\rho(t)}|(I-V)],
\end{aligned}
$$

with $V=U(t+\delta t) U^{\dagger}(t)$ and

$$
U(t)=T \exp \left(-\int_{0}^{t} \mathcal{A}(\mathrm{d} \rho / \mathrm{d} s) \mathrm{d} s\right),
$$

being the Uhlmann parallel transport unitary operator, in which $\mathcal{A}$ is the Uhlmann gauge field in the gauge provided by $\rho \mapsto \sqrt{\rho}$ (which is a global gauge for full rank density operators).

\section{Fidelity and Uhlmann connection in free fermion systems}

In our work, we applied the fidelity and $\Delta$ analysis of phase transitions on Boltzmann-Gibbs states 

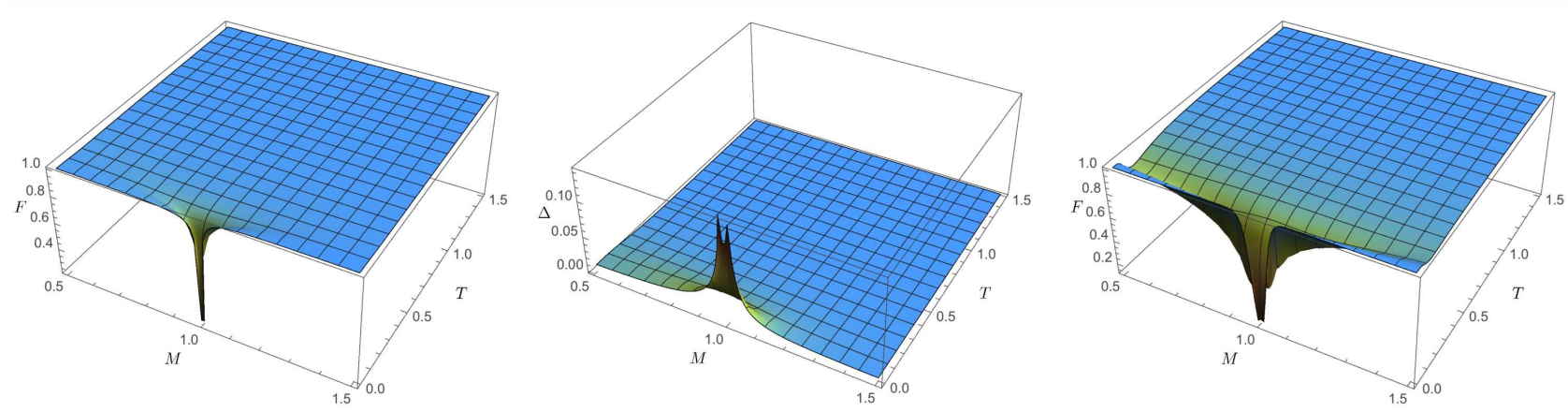

Fig. 2. From the left to the right: $F\left(\rho, \rho+\delta_{M} \rho\right)$ as a function of $T$ and $M, \Delta\left(\rho, \rho+\delta_{M} \rho\right)$ as a function of $T$ and $M$, $F\left(\rho, \rho+\delta_{T} \rho\right)$ as a function of $T$ and $M$. $\delta_{q} \rho$ denotes a variation of $\rho$ with respect to $q, q=M, T$. Here $\delta M=10^{-2}$ and $\delta T=10^{-2}$. The plot for $\Delta$ when deforming the state along $T$ is omitted because $\Delta$ is constant and equal to zero everywhere. (Adapted from Ref. [15]).

$$
\rho=\frac{\mathrm{e}^{-\beta \mathcal{H}}}{Z}
$$

where $\beta=1 / T$ is the inverse temperature, $Z$ is the partition function and $\mathcal{H}$ is a free-fermion Hamiltonian. The set of parameters is the coupling driving a quantum phase transition $(T=0)$ and the temperature $T$. We numerically evaluated $F\left(\rho, \rho^{\prime}\right)$ and $\Delta\left(\rho, \rho^{\prime}\right)$ with $\rho^{\prime}$ obtained from $\rho$ by a small deformation either on the coupling or on the temperature (or both). We analysed paradigmatic examples of topological insulators (the Creutz Ladder and the Su-Schrieffer-Heeger (SSH) model) and superconductors (Kitaev Chain) in dimension $D=1$ and also the BCS theory of superconductivity. Here we will focus on the Creutz Ladder and the BCS models. The results for the SSH and the Kitaev model are qualitatively similar to those of the Creutz Ladder model. Recently, we have also generalized the results obtained for topological phases of free fermions to $D=2$, with a further analytical proof of the absence of phase transitions at $T>0$ [17]. In particular, there we considered a Chern insulator and a topological superconductor.

\subsection{Creutz Ladder model}

The Creutz Ladder Hamiltonian is

$$
\begin{aligned}
\mathcal{H} & =-\sum_{i \in \mathbb{Z}}\left[K\left(\mathrm{e}^{-\mathrm{i} \phi} a_{i+1}^{\dagger} a_{i}+\mathrm{e}^{\mathrm{i} \phi} b_{i+1}^{\dagger} b_{i}\right)\right. \\
& \left.+K\left(b_{i+1}^{\dagger} a_{i}+a_{i+1}^{\dagger} b_{i}\right)+M a_{i}^{\dagger} b_{i}\right]+ \text { H.c. },
\end{aligned}
$$

where $K$ and $M$ are hopping amplitudes, the phase $\mathrm{e}^{\mathrm{i} \phi}$ is associated to a discrete gauge field and the $a_{i}, b_{i}$ 's are fermion annihilation operators for two different species at lattice sites specified by the Latin index $i$. For simplicity, we take $2 K=1$ and $\phi=\pi / 2$. Then, the system is in a non-trivial topological phase for $M<1$ and trivial for $M>1$. The phase of the system can be identified by going into momentum space where the Hamiltonian defines a map from the $1 D$ Brillouin zone, topologically a circle, to a great circle in the Bloch sphere. The winding number of this map modulo two determines the phase.
The results for the fidelity and $\Delta$ analysis are presented in Fig. 2. We see a clear change of the quantities in the vicinity of the zero-temperature quantum phase transition point. Clearly, we see no temperature driven phase transitions.

\subsection{BCS theory of superconductivity}

The BCS mean-field Hamiltonian is given by

$$
\begin{aligned}
\mathcal{H} & =\sum_{k}\left(\varepsilon_{k}-\mu\right)\left[\left(c_{k}^{\dagger} c_{k}-c_{-k} c_{-k}^{\dagger}\right)\right. \\
& \left.-\Delta_{k} c_{k}^{\dagger} c_{-k}^{\dagger}-\Delta_{k}^{*} c_{-k} c_{k}\right],
\end{aligned}
$$

where $c_{k} \equiv c_{k \uparrow}$ and $c_{-k} \equiv c_{-k \downarrow}$ are electron annihilation operators, the sum is over the momenta $k, \varepsilon_{k}$ is the spectrum, $\mu$ is the chemical potential and $\Delta_{k}$ is the superconducting gap. The latter is determined through selfconsistent equations and depends on the lattice-mediated pairing interaction denoted by $V$.

The results for the fidelity and $\Delta$ analysis are presented in Fig. 3.

To understand these results it is important to recall some facts about density matrices. Consider the Hilbert space $\mathbb{C}^{n}$. Then, density matrices are represented by Hermitian matrices. The latter can be diagonalized,

$$
\rho=S D S^{\dagger},
$$

where $S$ being a unitary matrix whose columns yield a basis of eigenvectors of $\rho$. Arbitrary deformations of $\rho$ can be written as follows:

$$
\delta \rho=S \delta D S^{\dagger}+\left[(\delta S) S^{\dagger}, \rho\right] .
$$

If the second term vanishes, only the spectrum is changing. If the first term vanishes, only the eigenspaces are changing.

In summary, fidelity captures phase transitions where both kinds of changes occur. The quantity $\Delta$ is capturing only deformations in eigenspaces. In contrast to BCS, no thermally driven phase transitions occurred in the Creutz Ladder model. The zero temperature phase transition features are washed out smoothly with temperature. Although the original Hamiltonian for BCS is not temperature dependent, the mean-field Hamiltonian is. 

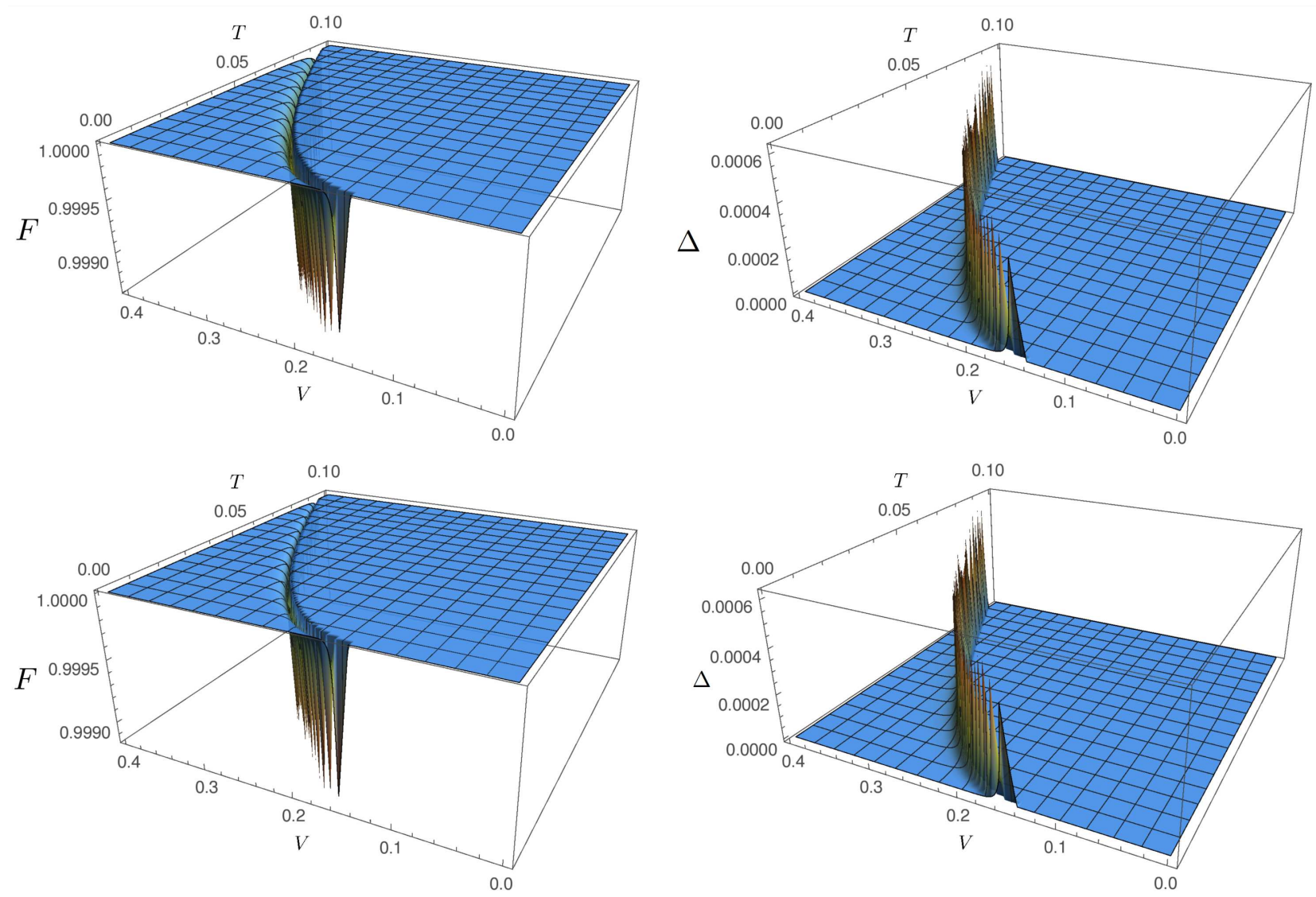

Fig. 3. From the left to the right, first row : $F\left(\rho, \rho+\delta_{V} \rho\right)$ as a function of $T$ and $M, \Delta\left(\rho, \rho+\delta_{V} \rho\right)$ as a function of $T$ and $M$. From the left to the right, second row : $F\left(\rho, \rho+\delta_{T} \rho\right)$ as a function of $T$ and $M, \Delta\left(\rho, \rho+\delta_{T} \rho\right)$ as a function of $T$ and $M . \delta_{q} \rho$ denotes a variation of $\rho$ with respect to $q, q=M, T$. Here $\delta T=10^{-3}$ and $\delta V=10^{-3}$. We clearly observe a temperature driven phase transition. (Adapted from Ref. [15]).

This shows that the origin of thermally driven phase transitions requires further investigation (see the Supplemental Material of Ref. [15]).

Previous studies [24] of the Uhlmann phase for the Creutz Ladder model where the space of parameters, i.e., the base space of the relevant bundle, was taken to be momentum and temperature indicated that a phase transition driven by temperature seemed to take place. In the paradigmatic case of the integer quantum Hall effect at $T=0$, we have that the transverse conductivity is given by the first Chern number of the occupied Bloch bundle. As a result, band topology appears in the response to an external field. It is unclear that the Uhlmann geometric phase along the one-dimensional momentum space, as a function of $T$, has an interpretation in terms of the response of the system. In contrast, the fidelity computed in our work, refers to the change of the system's overall state, with respect to its parameters (including temperature). Moreover, we have recently shown, by analysing the non-commutativity of the thermodynamic and zero temperature limits, that, in fact, there are no finite temperature phase transitions [17].

\section{Dynamical phase transitions: fidelity and interferometric Loschmidt echoes}

The real time evolution of closed quantum systems out of equilibrium has some formal similarities with thermal phase transitions. The non-analytic behaviour of certain dynamical observables after a sudden quench in a parameter of the Hamiltonian is now known as a dynamical phase transition. The analogy is made from the comparison

$$
\operatorname{Tr} \mathrm{e}^{-\beta H} \equiv \mathrm{e}^{-N f} \longleftrightarrow\left|\left\langle\psi\left|\mathrm{e}^{-\mathrm{i} t H}\right| \psi\right\rangle\right| \equiv \mathrm{e}^{-N g}
$$

where $N$ is the number of degrees of freedom of the system, $f$ is the free energy and $g$ is identified with the dynamical free energy. The function $g$ exhibits certain non-analytic behaviour as a function of $t$. When the quenched state becomes orthogonal to the initial state $g$ diverges - these are known as the Fisher zeroes.

\subsection{Figure of merit: Loschmidt echo}

Consider a smooth family of Hamiltonians parametrized by a smooth manifold $M,\{H(\lambda): \lambda \in M\}$. Take $\lambda_{i}, \lambda_{f} \in M$. The Loschmidt echo at time $t$ is the quantity 


$$
\mathcal{L}=\left|\left\langle\psi\left(\lambda_{i}\right)\left|\exp \left(-\mathrm{i} t H\left(\lambda_{f}\right)\right)\right| \psi\left(\lambda_{i}\right)\right\rangle\right|,
$$

where $|\psi(\lambda)\rangle$ is the groundstate of $H(\lambda)$ (assumed to be generically non-degenerate). As done in perturbation theory,

$$
\begin{aligned}
& \exp \left(-\mathrm{i} t H\left(\lambda_{f}\right)\right)= \\
& \quad \exp \left(-\mathrm{i} t H\left(\lambda_{i}\right)\right) T \exp \left(-\mathrm{i} \int_{0}^{t} \mathrm{~d} s V(s)\right),
\end{aligned}
$$

with $V(s)=\mathrm{e}^{\mathrm{i} s H\left(\lambda_{i}\right)}\left(H\left(\lambda_{f}\right)-H\left(\lambda_{i}\right)\right) \mathrm{e}^{-\mathrm{i} s H\left(\lambda_{i}\right)}$ being the "perturbation" in the interaction picture provided by $H\left(\lambda_{i}\right)$. It is then clear that

$$
\mathcal{L}=\left|\left\langle\psi\left(\lambda_{i}\right)\left|T \exp \left(-\mathrm{i} \int_{0}^{t} \mathrm{~d} s V(s)\right)\right| \psi\left(\lambda_{i}\right)\right\rangle\right| .
$$

To understand the relation with the quantum metric, we take $\lambda_{i} \equiv \lambda$ and $\lambda_{f}=\lambda+\delta \lambda$. Then,

$$
V=V_{a}(\lambda) \delta \lambda^{a}+\ldots, \text { with } V_{a}(\lambda)=\frac{\partial H}{\partial \lambda^{a}}(\lambda),
$$

where $\lambda^{a}$ are local coordinates on $M$ in a neighbourhood of $\lambda$. It is then a simple perturbation theory problem to show

$$
\begin{gathered}
\mathcal{L}=1-\frac{1}{2} \chi_{a b}(\lambda ; t) \delta \lambda^{a} \delta \lambda^{b}+\cdots \approx \\
\exp \left(-\frac{1}{2} \chi_{a b}(\lambda ; t) \delta \lambda^{a} \delta \lambda^{b}\right),
\end{gathered}
$$

with the dynamical susceptibility $\chi_{a b}(\lambda ; t)$ given by

$$
\begin{gathered}
\chi_{a b}(\lambda ; t)=\int_{0}^{t} \int_{0}^{t} \mathrm{~d} s_{2} \mathrm{~d} s_{1}\left(\left\langle V_{a}\left(s_{2}\right) V_{b}\left(s_{1}\right)\right\rangle\right. \\
\left.-\left\langle V_{a}\left(s_{2}\right)\right\rangle\left\langle V_{b}\left(s_{1}\right)\right\rangle\right),
\end{gathered}
$$

where $\langle\cdot\rangle:=\langle\psi(\lambda)|\cdot| \psi(\lambda)\rangle$.

Observe that $\mathcal{L}$ is nothing but the fidelity between the states $\left|\psi\left(\lambda_{i}\right)\right\rangle$ and $\mathrm{e}^{-\mathrm{i} t H\left(\lambda_{f}\right)}\left|\psi\left(\lambda_{i}\right)\right\rangle$. Recall that the fidelity $F$ is related to the Bures distance by $d_{B}^{2}=2(1-F)$. Hence, as we saw earlier, the quantum metric comes from the second order variation of the fidelity. Therefore, if we consider the family of maps $\Phi_{t}: \lambda^{\prime} \mapsto$ $\mathrm{e}^{-\mathrm{i} t H\left(\lambda^{\prime}\right)}|\psi(\lambda)\rangle\langle\psi(\lambda)| \mathrm{e}^{\mathrm{i} t H\left(\lambda^{\prime}\right)}$, take the pullback of the quantum metric and evaluate it at $\lambda^{\prime}=\lambda$, we obtain the dynamical susceptibility $\chi_{a b}(\lambda ; t)$.

\subsection{Generalizations: finite temperature}

One natural generalization is to take the fidelity between the states $\rho=\mathrm{e}^{-\beta H\left(\lambda_{i}\right)} / Z$ and the quenched state $\rho^{\prime}=\mathrm{e}^{-\mathrm{i} t H\left(\lambda_{f}\right)}\left(\mathrm{e}^{-\beta H\left(\lambda_{i}\right)} / Z\right) \mathrm{e}^{\mathrm{i} t H\left(\lambda_{f}\right)}$ - the $F i$ delity Loschmidt Echo. The associated susceptibility is then the pullback of the Bures metric in the space of full-rank density matrices by the map

$$
\Phi_{(t, \beta)}: \lambda^{\prime} \mapsto \mathrm{e}^{-\mathrm{i} t H\left(\lambda^{\prime}\right)}\left(\mathrm{e}^{-\beta H(\lambda)} / Z\right) \mathrm{e}^{-\mathrm{i} t H\left(\lambda^{\prime}\right)},
$$

evaluated at $\lambda^{\prime}=\lambda$.

Another possible generalization is constructed as follows. Recall the form of Eq. (45). If we replace the average on $|\psi(\lambda)\rangle$ by the average on $\mathrm{e}^{-\beta H(\lambda)} / Z$, the appropriate generalization is

$$
\begin{gathered}
\mathcal{L}=\left|\frac{\operatorname{Tr}\left\{\mathrm{e}^{-\beta H\left(\lambda_{i}\right)} \mathrm{e}^{\mathrm{i} t H\left(\lambda_{i}\right)} \mathrm{e}^{-\mathrm{i} t H\left(\lambda_{f}\right)}\right\}}{Z}\right|= \\
\left|\frac{\operatorname{Tr}\left\{\mathrm{e}^{-\beta H\left(\lambda_{i}\right)} T \exp \left(-\mathrm{i} \int_{0}^{t} \mathrm{~d} s V(s)\right)\right\}}{Z}\right| .
\end{gathered}
$$

We call it the Interferometric Loschmidt Echo. The associated susceptibility can also be seen as a pullback of a metric, but not in the space of states. Instead, it is the pullback of a metric in the space of unitary processes, namely, one induced by the thermal state $\rho(\lambda)=\exp (-\beta H(\lambda)) / Z$. This can be seen as a direct consequence of the following proposition: Let $A$ and $B$ be linear operators in the Hilbert space and a full rank density matrix $\rho$, then

$$
\langle A, B\rangle_{\rho} \equiv \operatorname{Tr}\left(\rho A^{\dagger} B\right)
$$

defines a Hermitian inner product. This induces a Riemannian metric in the space of unitary maps. The map by which we pullback to obtain the susceptibility is simply $\Phi_{t}: \lambda \mapsto \exp (-\mathrm{i} t H(\lambda))$. The interferometric Loschmidt echo has an interpretation in terms of an experiment. Consider the Mach-Zender interferometric experiment of Fig. 4.

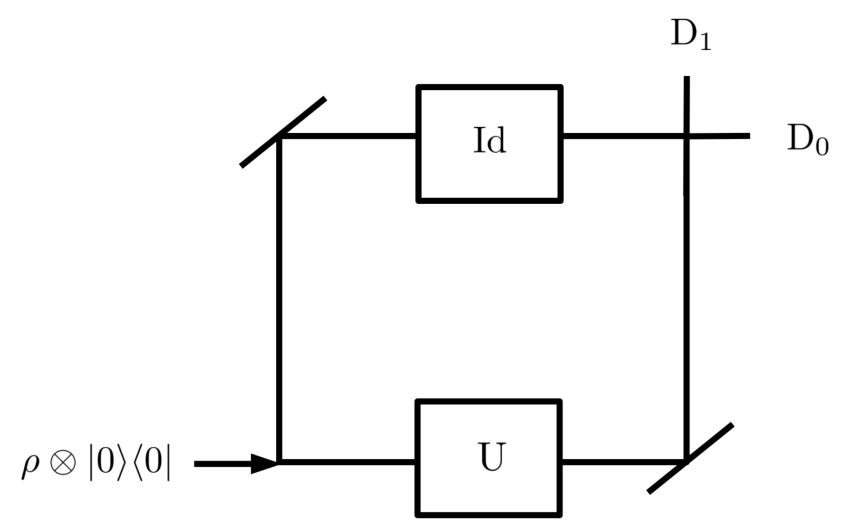

Fig. 4. Interferometric experiment associated to the interferometric Loschmidt echo.

The output in detector $D_{0}$ is obtained by measuring with respect to $\operatorname{Id} \otimes|0\rangle\langle 0|$, where the first factor space corresponds to the many-body state, and the second to the spatial degree of the system (the arms " 0 " and " 1 " of the interferometer), after acting on the input state with

$$
\begin{aligned}
& \mathrm{BS}_{2} \circ \mathrm{PS} \circ \mathrm{BS}_{1}= \\
& \frac{1}{\sqrt{2}}\left(\begin{array}{cc}
\mathrm{Id} & \mathrm{Id} \\
\mathrm{Id} & -\mathrm{Id}
\end{array}\right)\left(\begin{array}{cc}
\mathrm{Id} & 0 \\
0 & U
\end{array}\right) \frac{1}{\sqrt{2}}\left(\begin{array}{cc}
\mathrm{Id} & \mathrm{Id} \\
\mathrm{Id} & -\mathrm{Id}
\end{array}\right),
\end{aligned}
$$

where BS and PS stand for beam splitter and phase shift, respectively. The resulting sequence is 


$$
\begin{aligned}
\rho \otimes & |0\rangle\langle 0| \stackrel{\mathrm{BS}_{1}}{\longrightarrow} \frac{1}{2} \rho \otimes(|0\rangle\langle 0|+| 1\rangle\langle 1|+| 0\rangle\langle 1|+| 1\rangle\langle 0|) \\
& \stackrel{\mathrm{PS}}{\longrightarrow} \frac{1}{2}\left(\rho \otimes|0\rangle\left\langle 0\left|+U \rho U^{\dagger} \otimes\right| 1\right\rangle\left\langle 1\left|+\rho U^{\dagger} \otimes\right| 0\right\rangle\langle 1|\right. \\
& +U \rho \otimes|1\rangle\langle 0|) \\
& \stackrel{\mathrm{BS}_{2}}{\longrightarrow} \frac{1}{4}\left(\rho+U \rho U^{\dagger}+\rho U^{\dagger}+U \rho\right) \otimes|0\rangle\langle 0|+\ldots
\end{aligned}
$$

So, the output probability on $\mathrm{D}_{0}$ is

$$
\frac{1}{2}(1+\operatorname{Re} \operatorname{Tr}\{\rho U\}) .
$$

The second term has the same form as the interferometric Loschmidt echo, i.e., the trace of a density operator multiplied by a unitary operator.

\subsection{Results for two-band systems}

Take $H(\lambda)=\boldsymbol{x}(\lambda) \cdot \boldsymbol{\sigma}$ and with respect to a sphere of constant energy, i.e., $E=|\boldsymbol{x}|=$ constant, write

$$
\frac{\partial \boldsymbol{x}}{\partial \lambda^{a}}=\boldsymbol{t}_{a}+\boldsymbol{n}_{a}
$$

where the first term is tangent and the second is normal to the sphere. We then have that the dynamical fidelity susceptibility is given by

$$
\chi_{a b}=\tanh ^{2}(\beta E)\left(\frac{\sin (E t)}{E}\right)^{2} \boldsymbol{t}_{a} \cdot \boldsymbol{t}_{b},
$$

while the interferometric Loschmidt echo susceptibility is given by

$$
\begin{gathered}
\chi_{a b}=\left(\frac{\sin (E t)}{E}\right)^{2} \boldsymbol{t}_{a} \cdot \boldsymbol{t}_{b} \\
\quad+t^{2}\left(1-\tanh ^{2}(\beta E)\right) \boldsymbol{n}_{a} \cdot \boldsymbol{n}_{b} .
\end{gathered}
$$

Looking at the above formulae, we can conclude that for the fidelity Loschmidt echo, the dynamical susceptibility has no normal components. This is a direct consequence of the fact that the quenched density matrix has the same eigenvalues as the original one. For the case of the interferometric Loschmidt echo, the susceptibility has all types of components - the unitary process sees all the changes in $H$.

Intuitively, the interferometric Loschmidt echo is more sensitive than the Fidelity Loschmidt echo since it is associated with the details of the processes that lead to the final state, rather than the states themselves. However, the measurement of the former requires preparing Shrödinger cat-like states, which can be very challenging for many-body systems. The fidelity between two states corresponds to the classical fidelity after performing an optimal measurement on the two states.

The above formulae for the two-band case show that the fidelity susceptibility smears out with temperature, while the interferometric one does not, see Fig. 5. Moreover, in both cases, the shape function $\sin ^{2}(E t) / E^{2}$ is capturing the Fisher zeroes at critical times $t=(2 n+$ 1) $\pi / E$. We remark that the difference between the two gives a relation to thermal equilibrium:

$$
\left(1-\tanh ^{2}(\beta E)\right) \times\left[\left(\frac{\sin (E t)}{E}\right)^{2} \boldsymbol{t}_{a} \cdot \boldsymbol{t}_{b}+t^{2} \boldsymbol{n}_{a} \cdot \boldsymbol{n}_{b}\right],
$$

since the overall factor $\left(1-\tanh ^{2}(\beta E)\right)$ is the equilibrium susceptibility.

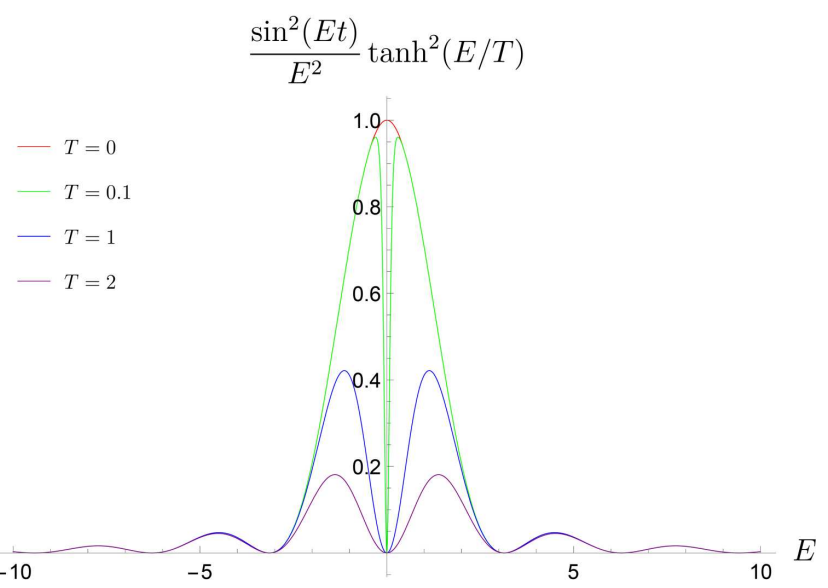

Fig. 5. Modulating function for the fidelity susceptibility. The gap closing points become less important with the increase of temperature. (Adapted from Ref. [18]).

\section{Conclusions}

In this work, we have presented a short overview of the information geometry approach to phase transitions. In particular, in the context of systems of free fermions at finite temperature, which are known exhibit topological phase transitions at zero temperatures, the BCS theory of superconductivity, and also in the context of dynamical phase transitions. We have seen, in all cases, that the topological features are gradually smeared out with temperature.

It is a remarkable fact that geometric quantities, such as the Bures metric, and the Berry and Uhlmann connections, are all linked to the quantum information concept of fidelity. The latter is able to capture all kinds of deformations of the state of the system. Ultimately, the intricate geometry and gauge theory arising in quantum mechanics is naturally associated to state distinguishability, which is captured by the fidelity.

\section{Acknowledgments}

The author greatly acknowledges C. Vlachou, N. Paunković, V.R. Vieira, O. Viyuela and S.T. Amin for the discussions and collaboration which made this work possible.

The author thanks the support from Fundação para a Ciência e Tecnologia (Portugal) namely through programmes $\mathrm{PTDC} / \mathrm{POPH} / \mathrm{POCH}$ and projects UID/EEA/50008/2013, UID/EEA/50008/2019, IT/QuSim, IT/QuNet, ProQuNet, partially funded by EU FEDER, from the EU FP7 project PAPETS 
(GA 323901) and from the JTF project NQuN (ID 60478). The author acknowledges the support from the project TheBlinQC supported by the EU H2020 QuantERA ERA-NET Cofund in Quantum Technologies and by FCT (QuantERA/0001/2017). The author also acknowledges the support of H2020 project SPARTA, projects QuantMining POCI-01-0145-FEDER-031826, PREDICT PTDC/CCI-CIF/29877/2017 and QBigData PEst-OE/EEI/LA0008/2013, by FCT.

\section{References}

[1] L.D. Landau, Ukr. J. Phys. 11, 19 (1937).

[2] P. Anderson, Basic Notions of Condensed Matter Physics, Westview Press, Boulder, CO, 1997.

[3] Y. Ando, J. Phys. Soc. Jpn. 82, 102001 (2013).

[4] D.J. Thouless, M. Kohmoto, M.P. Nightingale, M.D. Nijs, Phys. Rev. Lett. 49, 405 (1982).

[5] F.D.M. Haldane, Phys. Rev. Lett. 61, 2015 (1988).

[6] A. Kitaev, AIP Conf. Proc. 1134, 22 (2009).

[7] A.P. Schnyder, S. Ryu, A. Furusaki, A.W.W. Ludwig, Phys. Rev. B 78, 195125 (2008).

[8] A.P. Schnyder, S. Ryu, A. Furusaki, A.W.W. Ludwig, AIP Conf. Proc. 1134, 10 (2009).

[9] P. Zanardi, N. Paunković, Phys. Rev. E 74, 031123 (2006).

[10] N. Paunković, P.D. Sacramento, P. Nogueira, V.R. Vieira, V.K. Dugaev, Phys. Rev. A 77, 052302 (2008).
[11] D.F. Abasto, A. Hamma, P. Zanardi, Phys. Rev. A 78, 010301 (2008).

[12] P. Sacramento, B. Mera, N. Paunković, Ann. Phys. 401, 40 (2018).

[13] A. Uhlmann, Rep. Math. Phys. 24, 229 (1986).

[14] A. Uhlmann, Lett. Math. Phys. 21, 229 (1991).

[15] B. Mera, C. Vlachou, N. Paunković, V.R. Vieira, Phys. Rev. Lett. 119, 015702 (2017).

[16] B. Mera, C. Vlachou, N. Paunković, V.R. Vieira, J. Phys. A Math. Theor. 50, 365302 (2017).

[17] S.T. Amin, B. Mera, C. Vlachou, N. Paunković, V.R. Vieira, Phys. Rev. B 98, 245141 (2018).

[18] B. Mera, C. Vlachou, N. Paunković, V.R. Vieira, O. Viyuela, Phys. Rev. B 97, 094110 (2018).

[19] S. Morita, Geometry of Differential Forms, Vol. 201, American Mathematical Society, 2001.

[20] D. Chruscinski, A. Jamiolkowski, Geometric Phases in Classical and Quantum Mechanics, Vol. 36, Springer Science, 2012.

[21] M. Nakahara, Geometry, Topology and Physics CRC Press, 2003.

[22] N.E. Steenrod, The Topology of Fibre Bundles, Vol. 14, Princeton University Press, 1999.

[23] B. Mera, arXiv:1705.04394 (2017).

[24] O. Viyuela, A. Rivas, M.A. Martin-Delgado, Phys. Rev. Lett. 112, 130401 (2014). 